\title{
Psychological Wellbeing Facilitates Accurate HIV Risk Appraisal in Gay \& Bisexual Men
}

\author{
Rusi Jaspal \\ Nottingham Trent University, Nottingham, UK \\ Barbara Lopes \\ Universidade de Coimbra, Coimbra, Portugal
}

Background: Accurate HIV risk appraisal is key to effective HIV prevention. This study focuses on the role of psychological wellbeing in determining perceived HIV risk in a sample of gay and bisexual $(\mathrm{GBM})$ in the UK.

Methods: 191 HIV-negative GBM completed a cross-sectional survey, in which they provided demographic information and completed measures of actual engagement in actual sexual risk behaviours, diagnosis with an STI in the past 12 months, frequency of HIV testing, use of postexposure prophylaxis (PEP), perceived HIV risk, lesbian, gay, bisexual and transgender (LGBT) identity, sexual identity openness and psychological wellbeing.

Results: Results showed that there was a correlation between engagement in actual HIV risk behaviours and perceived HIV risk, suggesting relatively accurate HIV risk appraisal in the participant sample. LGBT identity and sexual identity openness were associated with increased psychological wellbeing. Structural equation modelling showed that psychological wellbeing has an effect on perceived HIV risk through the mediators of LGBT identity and sexual identity openness.

Conclusions: It appears that a state of psychological wellbeing facilitates the construction of a strong and robust LGBT identity, which can be displayed to others, and that a strong LGBT identity in turn facilitates accurate HIV risk appraisal in GBM. In order to achieve our target of zero new HIV infections by 2030, it will be essential to focus on enhancing psychological wellbeing in people at risk of HIV.

Keywords: HIV risk, risk appraisal, psychological wellbeing, identity, HIV prevention

\section{Citing this article}

Jaspal, R. Lopes, B. (in press). Psychological Wellbeing Facilitates Accurate HIV Risk Appraisal in Gay \& Bisexual Men. Sexual Health.

\section{Correspondence}

Professor Rusi Jaspal, School of Social Sciences, Nottingham Trent University, Nottingham, UK. Email: rusi.jaspal@cantab.net 


\section{Introduction}

Since the advent of effective antiretroviral therapy (ART), HIV has become a manageable chronic condition. However, ART can be accessed only if the individual is aware of their risk, decides to take an HIV test and engages with HIV care. In the UK, HIV disproportionately affects gay and bisexual men (GBM). ${ }^{1}$ Interventions to prevent HIV in this population tend to focus on enhancing awareness and understanding of HIV and its risk factors, increasing the acceptability of condom use and behaviour change. ${ }^{2}$ Behaviour change can occur only if the individual acknowledges their risk of HIV infection. Therefore, understanding why, and under what circumstances, GBM perceive themselves to be at risk of HIV is important. While there has been some research into the correlates of HIV risk perception, there is limited evidence concerning the contribution of psychological variables to HIV risk perception among GBM. Psychological wellbeing is relevant to GBM given that they face many social and psychological stressors, such as homophobia and decreased selfacceptance (in relation to sexual identity), which can undermine wellbeing. ${ }^{3}$ Thus, the present study focuses on the role of psychological wellbeing in determining perceived HIV risk in a sample of GBM in the UK.

\section{Psychological wellbeing and HIV risk behaviour}

Psychological wellbeing is defined as a state of acceptance of, and satisfaction with, one's present condition, and the construct encompasses individual wellness in relation to the psychological, social, mental, spiritual, physical and occupational dimensions of one's life. ${ }^{4}$ Furthermore, it refers to one's ability to utilise individual strengths (e.g., perseverance) to engage effectively with oneself, other individuals and social groups and to lead an authentic, meaningful and positive life. Psychological wellbeing is, thus, contingent on one's ability to construct a positive identity - characterised by selfesteem, distinctiveness, continuity and self-efficacy - and to display this identity to others. ${ }^{5}$ According to identity process theory from social psychology, ${ }^{6}$ adverse changes in one's social context (e.g. negative life events and experiences) can compromise psychological wellbeing and lead to 'threatened identity', which in turn gives rise to the deployment of coping strategies - these may be adaptive or maladaptive.

Consistent with identity process theory, there is an established relationship between decreased psychological wellbeing and HIV risk behaviour. ${ }^{7,8}$ Among GBM, there is a high prevalence of psychosocial stressors, such as childhood sexual abuse, homophobia, and internalised homophobia, which can undermine wellbeing. Childhood sexual abuse is up to four times more prevalent among GBM than heterosexual men and HIV-positive gay men are more likely than HIV-negative GBM to report a history of abuse. ${ }^{9}$ Having a history of abuse is in turn associated with engagement in HIV risk behaviours, possibly as a result of decreased self-esteem, self-care and self-efficacy. ${ }^{10}$ Furthermore, in their study of ethnic minority GBM, Jaspal et al. ${ }^{11}$ found that the relationship between childhood sexual abuse and HIV risk was mediated by substance misuse. It is possible that GBM who lack social support self-medicate against psychological adversity associated with childhood sexual abuse by engaging in the 'escapist' strategy of substance misuse. Substance misuse is itself a known predictor of HIV risk behaviour. ${ }^{8}$

Exposure to prejudice is related to HIV risk behaviours in GBM. ${ }^{12}$ Even in the UK, which has made significant strides in promoting LGBT rights and equality, many GBM report homophobia over the life course. ${ }^{3}$ Homophobia can result in internalised homophobia, that is, the uncritical acceptance and internalisation of homophobic stigma. ${ }^{13}$ This can preclude the construction of a strong gay identity. Due to the increased risk of threatened identity, life-long exposure to homophobia, and especially internalised homophobia, is associated with poor mental health outcomes, including anxiety, depression and suicidal ideation. ${ }^{14}$ These poor mental health outcomes predispose GBM to engage in HIV risk behaviours. ${ }^{8}$ In response to threats to psychological wellbeing, some GBM resort to maladaptive coping strategies, such as sexual compulsivity, drug use in sexualised settings, and unprotected anal intercourse. ${ }^{7,11}$ These behaviours may be responses to decreased psychological wellbeing but they do appear to contribute to HIV risk. Yet, these behaviours cannot be modified unless the individual has awareness of risk. 


\section{HIV risk perception}

Perceived HIV risk is important because, in most cases, it is the precursor to HIV testing, attempts to modify one's sexual behaviour and, if one is subsequently diagnosed with HIV, to access HIV care and ART. Several studies have shown that perceived HIV risk is a predictor of engagement with clinical services and with HIV prevention (e.g. pre-exposure prophylaxis), in that those who perceive themselves to be at either moderate or high risk of HIV infection are more likely to test for HIV and to express interest in PrEP. ${ }^{15,16,17}$ Perceived HIV risk can fluctuate over time in accordance with social contextual factors, such as relationships, behavioural 'phases', episodic engagement in substance use and sex work. ${ }^{18}$

HIV risk perception is in part determined by social psychological variables. For instance, empirical research shows that people tend to 'other' HIV and perceive it as affecting outgroups or people unlike themselves. ${ }^{19}$ Moreover, there is some evidence that the desire to 'resist' stigma can lead individuals to downplay their own risk of HIV infection irrespective of their objective risk. ${ }^{20,21}$ Goldenberg et al. ${ }^{22}$ reported that interviewees perceived sexual intercourse with partners who did not identify as gay, who were not out or who also had sex with women as being 'low risk'. These men consequently perceived themselves to be at low risk of HIV. A survey study of GBM in Australia revealed that over $75 \%$ of participants believed that people living with HIV should disclose their HIV status before sex and therefore assumed that their partners were all HIV-negative. ${ }^{23}$ They therefore perceived their own risk of HIV to be low.

In their study of high-risk heterosexual people in the US, Koku and Felsher ${ }^{24}$ found that risk perception was contingent on interpersonal relationships - perceived HIV risk of partners and racial ingroup members, density of drug ties, sex within relationships and emotional closeness within relationships all predicted perceived HIV risk. Furthermore, Stephenson et al. ${ }^{25}$ provide further evidence that relationship type influences HIV risk perception - in their study of 906 gay men, they found that those in open relationships were more likely than those in monogamous relationships to perceive themselves to be at risk of HIV infection.

Several studies suggest that HIV risk perception among GBM is often inaccurate. For instance, Jaspal et al. ${ }^{26}$ found that those participants who perceived their sexual behaviour to be least risky also scored the lowest on HIV knowledge, suggesting that their HIV risk appraisal is based on an inadequate understanding of HIV. Furthermore, Australia, Mao et al. ${ }^{27}$ found that participants who reported condomless anal sex with a casual partner were less likely to perceive themselves to be at risk of HIV than those who reported consistent condom use in the last six months. A survey of 267 young gay men in six US cities revealed that half of those who had undiagnosed HIV perceived their lifetime risk of infection to be low. ${ }^{28}$ Sarno $^{29}$ found that physical attractiveness of a potential sexual partner was negatively associated with perceived HIV risk. In other words, individuals perceived a physically attractive partner as less likely to be HIV-infected, using this as the principal mechanism for appraising their own risk of infection. Although these studies suggest inaccurate risk appraisal, the increased focus on enhancing awareness and understanding of HIV risk in GBM over several years is likely to have resulted in more accurate HIV appraisal in GBM.

Existing empirical research into HIV risk perception tends to focus on interpersonal and intergroup, rather than psychological factors. Only empirical research into actual HIV risk behaviour acknowledges the role of psychological wellbeing. Conversely, the focus of the present study is on the specific variable of psychological wellbeing in perceived HIV risk.

\section{Hypotheses}

In this study, the following hypotheses are tested:

1. In view of growing awareness and understanding of HIV among GBM, there will be a correlation between engagement in actual HIV risk behaviours and perceived HIV risk.

2. Consistent with identity process theory, a stronger LGBT identity and greater sexual identity openness will be associated with increased psychological wellbeing.

3. Psychological wellbeing will have an effect on perceived HIV risk through the mediators of 
LGBT identity and sexual identity openness.

\begin{abstract}
Methods
Ethics

The Health and Life Sciences Faculty Research Ethics Committee at De Montfort University, Leicester, UK provided ethics approval for this study, which was conducted in accordance with the British Psychological Society Code of Ethics and Conduct.
\end{abstract}

\title{
Participant recruitment
}

191 HIV-negative GBM were recruited on Grindr® a geospatial mobile gay social networking application in July 2017. 191 (82\%) of the 234 users who initiated the survey completed it and, thus, 191 cases were included in the analysis. Only Grindr users in the British city of Leicester were targeted to participate in the study.

\section{Measures}

Participants completed a survey with questions focusing on various demographic factors (e.g. age, ethnicity, education level, income), actual engagement in actual sexual risk behaviours, diagnosis with an STI in the past 12 months, frequency of HIV testing, use of post-exposure prophylaxis (PEP), perceived HIV risk, LGBT identity, sexual identity openness and psychological wellbeing. Frequency of engagement in actual sexual risk behaviour was measured by computing the scores of 3 items that measured the frequency of cruising, using geospatial applications, and using gay saunas to find sexual partners. The scale included items such as "In the past month, how often have you had sex with other men you met on the Internet or a mobile application (e.g. Grindr)?".

Responses were on a Likert scale from $1=$ never to $6=$ everyday. The scale manifested acceptable reliability, $\alpha=.59$. Perceived HIV risk was measured using the HIV Perceived Risk Scale, ${ }^{30}$ which consists of 8 items and includes items such as "There is a chance, no matter how small, I could get HIV". Respondents indicated the extent of their agreement with statements on a Likert scale from $1=$ strongly disagree to $5=$ strongly agree. Higher scores indicate more perceived HIV risk. The scale had good reliability, $\alpha=76$. Diagnosis with an STI in the past 12 months was measured using the item "In the last 12 months, have you been diagnosed with any sexually transmitted infections (STIs)?", which was dummy coded for analysis. Frequency of HIV testing was measured using the item "How often do you get tested for HIV?" Responses were on a Likert scale from 1=every 3 months to $5=$ never. $\boldsymbol{L G B T}$ identity was measured using the Lesbian, Gay and Bisexual Identity Scale. ${ }^{31}$ The scale consists of 12 items such as "Being gay or bisexual is very important to my sense of who I am". Responses were on a Likert scale from 1= strongly disagree to 5= strongly agree. Higher scores indicate a stronger LGBT identity. The scale had acceptable reliability $\alpha=.57$. Sexual identity openness was measured using the item "How open are you to other people about your sexual orientation?" Responses were on a Likert scale from $1=$ not at all open to $5=$ very open. Psychological wellbeing was measured using the Life Satisfaction Scale, ${ }^{32}$ which consists of 7 items such as "I've been dealing with problems well". Responses were on a Likert scale from $1=$ strongly disagree to $5=$ strongly agree. Higher scores indicate more wellbeing. The scale had very good reliability, $\alpha=.90$.

\section{Data analysis}

SPSS and AMOS version 20 were used to perform the analyses. Given that most variables of interest were not normally distributed, non-parametric Kruskal-Wallis tests and Spearman Rho's correlations were conducted using SPSS to test hypotheses 1 and 2. Kruskal-Wallis tests analyse differences between groups in the sample (sexual orientation; religion; and relationship status) for the key variables. Structural equation modelling on AMOS version 20 was used to test hypothesis 3 . Transformations were applied to all nonnormally distributed variables, resulting in normal distributions, and a bootstrap at 200 samples was used. Assumptions of linearity (normal probability plot), homoscedasticity (plot of residuals versus predicted value), independence (Durbin-Watson 
statistic) of residuals, the presence of outliers (Cook's distance <1 except for one value) and multicollinearity (Variance Inflation Factor $(\mathrm{VIF})<2$ ) were also tested and no major problems were found.

\section{Descriptive statistics on participant sample}

\section{Results}

Participants had a mean age of 33.6 years $(\mathrm{SD}=11.2)$. In terms of ethnicity, $141(74 \%)$ participants described themselves as White British; 26 (9\%) as South Asian; and $24(8 \%)$ as White Other. Of the 191 participants, 111 reported no religion (58\%); 54 reported being Christian (28\%); 6 reported being Hindu (6\%); 10 reported being Muslim (10\%); 2 reported being Sikhs (1\%) and 6 reported 'Other' as their religion (3\%). 141 (74\%) participants self-identified as gay and $43(23 \%)$ as bisexual. $22 \%$ of participants reported an income of $\langle £ 10,000 ; 37 \% £ 10,000-24,999 ; 18 \% £ 25,000-34,999 ; 8 \%$ $£ 35,000-44,999 ; 6 \% £ 45,000-54,999$ and $6 \%>£ 55,000$. Thirty-three (17\%) participants reported GCSE/O-level education; 49 (26\%) reported having A-level education (26\%); 45 (24\%) reported having a university degree; $46(24 \%)$ reported having postgraduate education; and $17(9 \%)$ another type of education. Of the 191 respondents, $114(60 \%)$ were single; $38(20 \%)$ were in a monogamous relationship; $20(11 \%)$ were in an open relationship; $15(8 \%)$ were married; $1(.5 \%)$ was in a civil partnership; and 3 (2\%) were in other types of relationship.

\section{Tests of normality}

Kolmogorov-Smirnov tests showed that the following variables were not normally distributed: frequency of engagement in actual sexual risk behaviour $(D(184)=3.86, p<.001)$; PEP use $(D$ $(184)=7.16, p<.001)$; frequency of HIV testing $(D(184)=-3.24, p=.001)$; STI diagnoses in the past 12 months $(D(181)=7.04, p<.001)$; sexual identity openness $(D(191)=2.83, p<.001)$; and psychological wellbeing $(D(161)=1.38, p=.045)$.

\section{[Insert Table 1 about here]}

\section{Differences between sexual orientation}

Kruskal-Wallis tests showed that there were no statistically significant differences between sexual orientation groups (gay, bisexual and other) for psychological wellbeing $H(3)=3.70, p=.30$ ); LGBT identity $(H(3)=5.51, p=.14)$; perceived HIV risk $(H(3)=3.22, p=.36)$; frequency of engagement in actual sexual risk behaviour $(H(3)=1.86, p=.60)$; and frequency of HIV testing $(H \quad(3)=6.81, p=.076)$. Therefore, all subsequent analyses were performed with all 191 individuals.

\section{Effect of religion on key variables}

Kruskal Wallis tests showed that religion had an effect only on sexual identity openness $(H(5)=25.07$, $p<.001)$, and on no other variable. Participants who reported no religion $(M=2.23, S D=1.20)$, being Christian $(M=2.24, S D=1.12)$ or 'Other' religion $(M=1.83, S D=.75)$ reported more sexual identity openness than Hindus $(M=4.17, S D=.04)$, Muslims $(M=3.50, S D=1.13)$ and Sikhs $(M=4.50, S D=.070)$.

\section{Effect of relationship status on key variables}

Kruskal Wallis tests showed that relationship status had an effect on sexual identity openness $(H$ $(5)=11.67, p=.040)$; LGBT identity $(H(5)=17.01, p=.004)$; frequency of actual sexual risk behaviour $(H(5)=24.07, p<.001)$; and perceived HIV risk $(H(5)=21.20, p=.001)$.

Participants in a monogamous relationship $(M=1.87, S D=.94)$ exhibited more sexual identity openness than people who were single $(M=2.56, S D=1.28)$; married $(M=2.67, S D=1.40)$; and in an open relationship $(M=2.10, S D=1.12)$.

Those in a monogamous relationship reported a stronger LGBT identity $(M=41.34, S D=5.97)$ than those who were single $(M=36.04, S D=9.07)$; married $(M=37.45, S D=6.14)$ and in an open relationship $(M=39.34, S D=5.97)$.

Participants in a monogamous relationship and married participants reported less frequent 
engagement in actual sexual risk behaviour $(M=3.44, S D=1.11$ and $M=3.73, S D=.088$, respectively) than those in an open relationship $(M=4.53, S D=1.47)$, in other types of relationship $(M=4.33$, $S D=1.53)$ and single $(M=4.64, S D=2.16)$.

Respondents in a monogamous relationship reported less perceived HIV risk $(M=18.47$, $S D=4.5)$ than people who are single $(M=23.15, S D=4.65)$; in an open relationship $(M=23.39$, $S D=4.65)$; and married $(M=22.50, S D=5.67)$.

\section{Correlations}

Psychological wellbeing was positively correlated with LGBT identity, meaning that the more individuals accepted their LGBT identity the better their psychological wellbeing was $(r=.36, p<.001)$. Also, the more participants accepted their LGBT identity, the more open they were about their sexual identity $(r=.43, p<.001)$ and the less they perceived HIV risk to be ( $r=.-18, p=.030)$. This supports the hypothesis that a stable and accepting LGBT identity is associated with more psychological wellbeing and more openness about sexual identity.

Frequency of engagement in actual risk behaviour was positively associated with perceived HIV risk $(r=.44, p<.001)$, which suggested that participants were aware that engaging in these behaviours is associated with increased HIV risk. Moreover, frequency of engagement in actual risk behaviour was positively associated with an STI diagnosis in the past 12 months $(r=.24, p=.001)$, suggesting that these behaviours are actually associated with the hazard (that is, STIs). Perceived HIV risk was related to frequency of HIV testing $(r=.21, p=.008)$. Frequent HIV testing in turn was also positively correlated with frequency of engagement in sexual risk behaviours $(r=.28, p=.001)$. These findings support the hypothesis that there is an association between engagement in actual HIV risk behaviours and perceived HIV risk.

\section{[Insert Figure 1 about here]}

\section{Structural equation model}

A structural equation model was conducted with AMOS version 20 and was constructed with psychological wellbeing as a predictor; perceived HIV risk as the outcome variable; and LGBT identity and sexual identity openness as mediators of the relationship between wellbeing and perceived HIV risk. The model (Figure 1) was statistically significant $\chi^{2}=5.054(d f=0), p<.001$. Comparative Fit index (CFI) was 0.99; Tucker Lewis Index(TLI) was 0.98 and Root Square Mean error (RMSEA) <.05 suggesting a good fit. Only indirect paths were included.

As predicted, psychological wellbeing significantly predicted the variance of LGBT identity with a $\beta=.36, \mathrm{SE}=.102$, Bootstrap 95\% CI $(.305,710), p<.001$, and LGBT identity in turn emerged as a statistically significant mediator of the effect of psychological wellbeing on HIV risk with a $\beta$ $=-.23, \mathrm{SE}=.052$, Bootstrap 95\% CI $(-.235,-.035), p=.008$. Thus, LGBT identity statistically significantly mediates the effect of psychological wellbeing on perceived HIV risk. This suggests that a state of psychological wellbeing facilitates an LGBT identity, which in turn enables the gay or bisexual individual to perceive his risk of HIV.

As expected, LGBT identity also had a statistically significant effect on sexual identity openness, with a $\beta=.42, \mathrm{SE}=.003$, Bootstrap 95\% CI (-.028, -.014), $p<.001$, suggesting that having a strong LGBT identity was a precursor to displaying this to other people. Furthermore, sexual identity openness did not have an effect on perceived HIV risk with a $\beta=.022, \mathrm{SE}=1.165$ Bootstrap 95\% CI $(-2.082,2,451), p=.79$, suggesting a greater psychological, rather than interpersonal, antecedent to HIV risk perception. This partially supports the hypothesis that psychological wellbeing has an effect on perceived HIV risk through the mediators of LGBT identity and sexual identity openness.

\section{Discussion}

It is important to understand not only the social psychological predictors of actual engagement in HIV risk behaviour but also of perceived HIV risk. There is overwhelming evidence that perceived HIV risk is associated with engagement with prevention strategies, such as condoms and PrEP, and with 
HIV testing. Increased engagement with HIV prevention, testing and care at a population level will in turn enable us to meet the ambitious target of zero new HIV transmissions by $2030 .{ }^{1}$ The present study shows that, in addition to various interpersonal factors identified in previous empirical research, psychological wellbeing is an important predictor of HIV risk perception. Moreover, in our sample at least, perceived HIV risk appears to be broadly consistent with actual HIV risk behaviour. Thus, in contrast to earlier empirical research, HIV risk appraisals may be improving in GBM.

A key finding from this study is that a state of psychological wellbeing facilitates the construction of a strong and robust LGBT identity, that is, self-construal as gay or bisexual and identification with the sexual ingroup. In short, feeling psychologically well enables the individual to derive a positive self-conception and to relate effectively to other people on the basis of their sexual orientation. Conversely, the inability to construct such an identity despite same-sex attraction may be associated with decreased psychological coherence, on the one hand, and reflect internalised homophobia, on the other hand. Psychological coherence refers to the psychological need for perceived compatibility and coherence between elements of identity. ${ }^{33}$ Internalised homophobia refers to GBM's uncritical acceptance and internalisation of the homophobia and stigma that appears to be pervasive, though subtly expressed, in society. ${ }^{14}$ Both threatened psychological coherence and internalised homophobia are associated with actual engagement in HIV risk behaviour. ${ }^{7}$ It is possible that a strong LGBT identity enables GBM to acknowledge aspects of their sexual identity and, thus, the reality of their risk behaviour. This may in turn facilitate adequate engagement with prevention and care and less proneness to engage in sexual risky behaviours.

The study also revealed a relationship between a strong LGBT identity and sexual identity openness, that is, the ability and willingness to display this identity to other people. Although we did not find a significant relationship between sexual identity openness and HIV risk perception, it is possible that by being more open about their sexual identity, GBM find themselves in a better position to access information about sexual health and HIV and, thus, to take steps to modify their sexual behaviour to reduce their risk of infection. However, this relationship must be verified in future empirical research.

Moreover, it is likely that a bidirectional relationship exists between sexual identity openness and psychological wellbeing, which, according to our findings, does appear to be central to perceived HIV risk. This can be attributed to the increased likelihood of identity authenticity, which is important for psychological wellbeing, ${ }^{34}$ and of exposure to validating messages about one's sexual orientation through association with other GBM. ${ }^{35}$ Exposure to validating messages about an immutable aspect of one's identity, such as sexual orientation, is likely to enhance psychological wellbeing. Clearly, it is necessary to be open about one's sexual identity - at least to some people - in order to access such validating messages. Decreased sexual identity openness might conversely facilitate entrenched internalised homophobia and lead GBM to 'suffer in silence' without recourse to social support. Yet, social context is key and GBM can expect to access validating messages in social contexts in which their sexual orientation is valued and respected. In societies with high levels of homophobia - even state-sanctioned homophobia - it is difficult to see how sexual identity openness would be conducive to psychological wellbeing. ${ }^{36}$

Our findings are broadly consistent with the 'cognitive miser' approach in social cognition research, which suggests that people are economically prudent in cognition and thus think in less effortful, rather than rational, ways to solve problems. ${ }^{37}$ When individuals are cognitively focused on resisting the 'threat' of stigma and other stressors, they may be less inclined to see the broader picture and, thus, fail to appraise their risk in a rational manner. ${ }^{38}$ It could be hypothesised that GBM with compromised psychological wellbeing invest their cognitive 'energy' in addressing their wellbeing issues, rather than in acknowledging their risk of HIV infection and in taking effective steps to reduce their risk. Evidently, this hypothesis will need to be examined in future research. Furthermore, to acknowledge their risk of HIV could further contribute to the stigma that many GBM

${ }^{1}$ https://www.hivcommission.org.uk/2019/07/22/announcement-press-release/ 
face and internalise. This is also consistent with the notion that acknowledgement of risk is psychologically threatening and that risk may therefore be resisted or denied for the protection of identity and psychological wellbeing. ${ }^{39}$

GBM continue to face a multitude of social and psychological stressors which increase the risk of decreased psychological wellbeing and which undermine the construction of a robust LGBT identity about which they can be open to others. These include inter alia poor mental health, homelessness, childhood sexual abuse, homophobia and internalised homophobia. ${ }^{40,8}$. There is clearly a need for continued efforts to address these social psychological stressors and the strategies for doing so will necessarily be multifarious and multisectoral.

The high prevalence of homophobia and stigma due to sexual orientation, which is reported by GBM, lies at the heart of many of these stressors and must be challenged through effective public awareness-raising campaigns. This demonstrates the importance of inclusive sex education offered in schools and colleges, which can disseminate the message to both GBM and to the general population that sexual diversity is to be celebrated rather than denigrated. Furthermore, efforts to address homophobia in ethnic and religious minority communities will be especially important given the greatest challenges appear to lie in these communities and are faced by GBM who identify with them. ${ }^{34}$ Our data suggest that psychological wellbeing, as a predictor perceived HIV risk, is important for many reasons - not least HIV prevention and, thus, public health.

\section{Limitations and future directions}

Although previous empirical research has examined the effect of homophobia, internalised homophobia, childhood sexual abuse and internalised homophobia on psychological wellbeing and HIV risk behaviour, our study did not include measures of these specific variables. In future research, effort should be made to explore the pathways between these social psychological stressors, wellbeing, LGBT identity and HIV risk appraisal in GBM.

Moreover, the present study focuses on GBM - most of whom were of White British ethnicity - in a just one city in the UK. It is hoped that future studies will replicate these findings in other geographical contexts and with other populations - especially those at particularly high risk of HIV, such as women, ethnic minority individuals, and transgender people. It is also important to replicate this study in other countries, in which the social and psychological 'stressors' experienced by GBM may be greater or qualitatively different. For instance, in societies in which GBM experience high levels of prejudice due to their sexual orientation, ${ }^{36,41,42}$ it is possible that there are greater levels of sexual identity concealment, which in turn may have an effect on their HIV risk perception. Further research in other contexts will facilitate the design of appropriate behavioural interventions for the prevention of HIV for specific subgroups of GBM and potentially other populations.

\section{Conclusions}

In view of these preliminary findings, it can be argued that psychological wellbeing in GBM is conducive to a strong LGBT identity, and vice-versa, and that this is associated with more accurate HIV risk appraisals. Thus, for us to achieve our target of zero new HIV infections by 2030, it will be essential to focus on enhancing psychological wellbeing in this key population - our enormous advances in HIV science must be coupled with interventions to enhance psychological wellbeing in a population vulnerable to social and psychological stressors.

\section{Funding statement}

This research did not receive any specific funding.

\section{Conflicts of interest}

The authors declare no conflicts of interest. 
Table 1. Descriptive statistics for the main variables and socio-economic and demographic characteristics of the participant sample

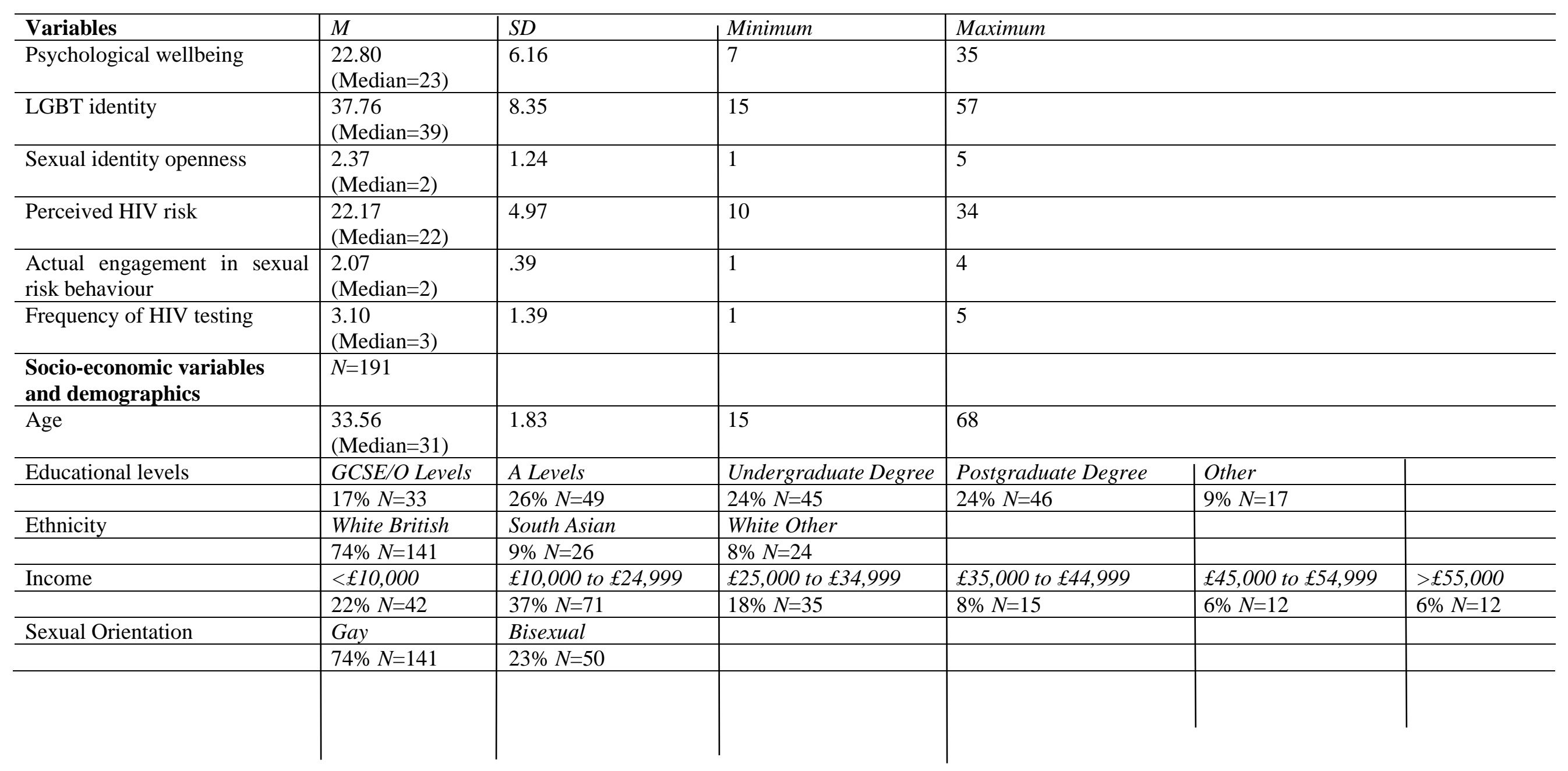


Figure 1. Structural equation model with psychological wellbeing predicting perceived HIV risk through LGBT identity and sexual identity openness.

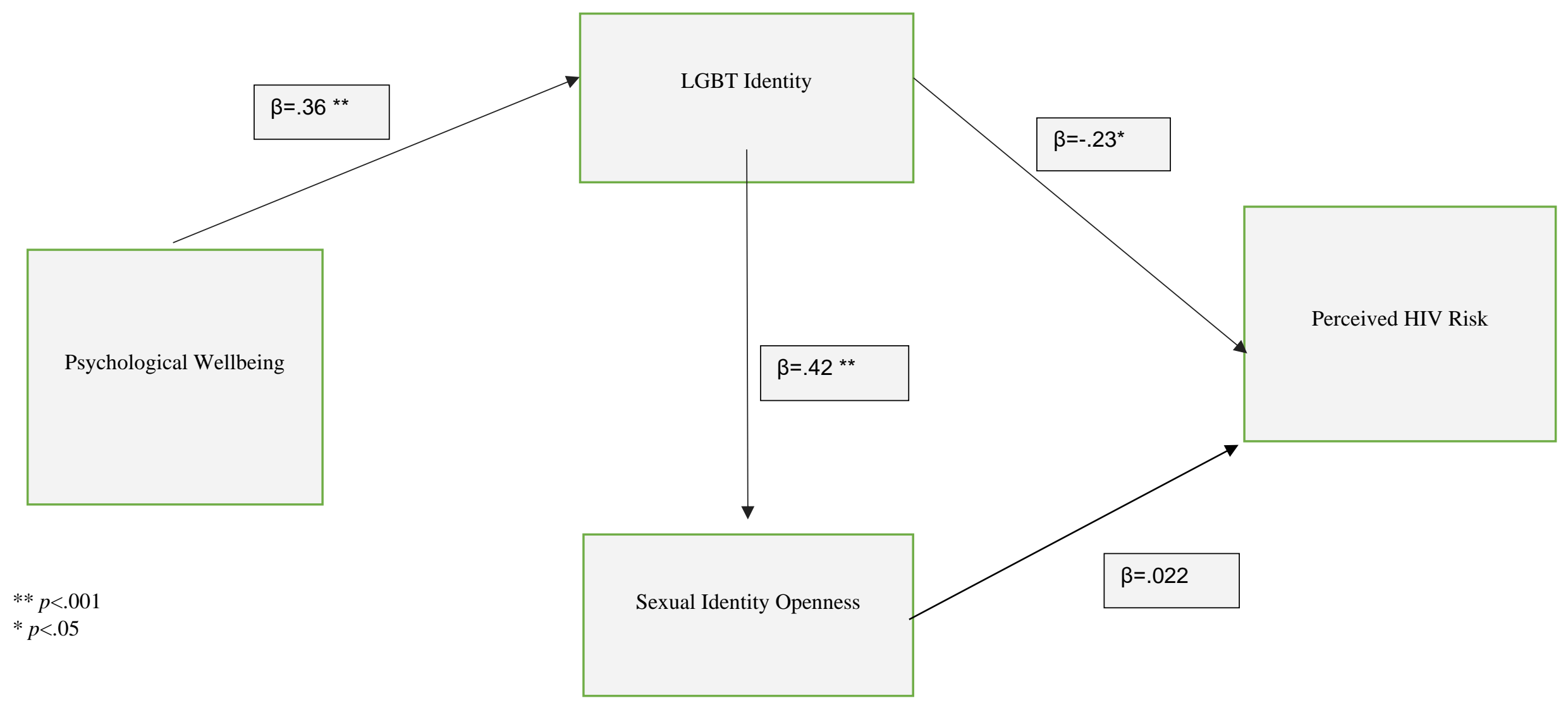




\section{References}

${ }^{1}$ Public Health England. Progress towards ending the HIV epidemic in the United Kingdom. London: Public Health England; 2017. Available online at https://assets.publishing.service.gov.uk/government/uploads/system/uploads/attachment_data/file/8 21273/Progress towards ending the HIV epidemic in the UK.pdf

[verified 14 September 2018].

${ }^{2}$ Fish J, Papaloukas P, Jaspal R, Williamson I. Equality in sexual health promotion: a systematic review of effective interventions for black and minority ethnic men who have sex with men. BMC Public Health 2016; 16(810). doi:10.1186/s12889-016-3418-x

${ }^{3}$ Jaspal R. The social psychology of gay men. London: Palgrave; 2019.

${ }^{4}$ Seligman MEP. Flourish: a visionary new understanding of happiness and well-being. New York: Free Press; 2011.

${ }^{5}$ Breakwell GM. Coping with threatened identities. London: Methuen; 1986.

${ }^{6}$ Jaspal R, Breakwell GM. eds. Identity process theory: identity, social action and social change. Cambridge: Cambridge University Press; 2014.

${ }^{7}$ Jaspal R. Enhancing sexual health, self-Identity and wellbeing among men who have sex with men: a guide for practitioners. London: Jessica Kingsley Publishers; 2018.

${ }^{8}$ Safren SA, Reisner SL, Herrick A, Mimiaga MJ, Stall RD. Mental health and HIV risk in men who have sex with men. Journal of Acquired Immune Deficiency Syndromes 2010; 55(Suppl 2): S74-S77. doi:10.1097/QAI.0b013e3181fbc939

${ }^{9}$ Lloyd S, Operario D. HIV risk among men who have sex with men who have experienced childhood sexual abuse: systematic review and meta-analysis. AIDS Education \& Prevention 2012; 24(3): 228-41. doi:10.1521/aeap.2012.24.3.228.

${ }^{10}$ Rosario M, Schrimshaw EW, Hunter J. A model of sexual risk behaviors among young gay and bisexual men: longitudinal associations of mental health, substance abuse, sexual abuse, and the coming-out process. AIDS Education \& Prevention 2006; 18(5): 444-60.

doi:10.1521/aeap.2006.18.5.444

11 Jaspal R, Lopes B, Jamal Z, Paccoud I, Sekhon P. Sexual abuse and HIV risk behaviour among black and minority ethnic men who have sex with men in the UK. Mental Health, Religion \& Culture 2017; 20(8): 841-53. doi:10.1080/13674676.2017.1414170

12 Díaz RM, Ayala G, Bein E. Sexual risk as an outcome of social oppression: data from a probability sample of Latino gay men in three U.S. cities. Cultural Diversity and Ethnic Minority Psychology 2004; 10(3): 255-267. doi:10.1037/1099-9809.10.3.255

${ }_{13}$ Williamson IR. Internalized homophobia and health issues affecting lesbians and gay men. Health Education Research 2000; 15(1): 97-107. doi:10.1093/her/15.1.97

${ }^{14}$ Walch SE, Ngamake ST, Bovornusvakool W, Walker SV. Discrimination, internalized homophobia, and concealment in sexual minority physical and mental health. Psychology of Sexual Orientation and Gender Diversity 2016; 3(1): 37-48. doi:10.1037/sgd0000146

${ }^{15}$ Biello KB, Edeza A, Montgomery MC, Almonte A, Chan PA. Risk perception and interest in HIV pre-exposure prophylaxis among men who have sex with men with rectal gonorrhea and chlamydia infection. Archives of Sexual Behavior 2019; 48(4): 1185-1190. doi:10.1007/s10508018-1260-1

${ }^{16}$ Kahle EM, Sharma A, Sullivan SP, Stephenson R. HIV prioritization and risk perception among an online sample of men who have sex with men in the United States. American Journal of Men's Health 2018; 12(4): 676-687. doi:10.1177/1557988318776581

${ }^{17}$ Jaspal R, Lopes B, Bayley J, Papaloukas P. A structural equation model to predict the acceptability of pre-exposure prophylaxis in men who have sex with men in Leicester, UK. HIV Medicine 2019; 20(1): 11-18. doi:10.1111/hiv.12667

${ }^{18}$ Underhill K, Guthrie KM, Colleran C, Calabrese SK, Operario D, Mayer KH. Temporal 
fluctuations in behavior, perceived HIV risk, and willingness to use pre-exposure prophylaxis (PrEP). Archives of Sexual Behavior 2018; 47(7): 2109-2121. doi:10.1007/s10508-017-1100-8

19 Joffe H. Identity, self-control, and risk. In Moloney G, Walker I, editors. Social representations and identity. New York: Palgrave Macmillan; 2007. pp. 197-213.

${ }^{20}$ Baros S, Grujicic SS, Zikic B, Atay JP. Stigma matters: HIV and HIV risk perception among men who have sex with men in Serbia; a qualitative study. Health, Risk \& Society 2018; 20(78): 342-357. doi:10.1080/13698575.2018.1551991

${ }^{21}$ Jaspal R, Daramilas C. Perceptions of pre-exposure prophylaxis (PrEP) among HIV-negative and HIV-positive men who have sex with men. Cogent Medicine 2016; 3: 1256850.

doi:10.1080/2331205X.2016.1256850

${ }^{22}$ Goldenberg T, Finneran C, Sullivan SP, Andes KL, Stephenson R. "I consider being gay a very high risk factor": How perceptions of a partner's sexual identity influence perceptions of HIV risk among gay and bisexual men. Sexuality Research and Social Policy 2017; 14(1): 32-41. doi:10.1007/s13178-016-0235-z

${ }^{23}$ Murphy DA, de Wit JB, Donohoe S, Adam PC. The need to know: HIV status disclosure expectations and practices among non-HIV-positive gay and bisexual men in Australia. AIDS Care 2015; 27(Suppl 1): 90-98. doi:10.1080/09540121.2015.1062077

${ }^{24}$ Koku E, Felsher M. The effect of social networks and social constructions on HIV risk perceptions. AIDS and Behavior 2019: doi:10.1007/s10461-019-02637-y.

${ }^{25}$ Stephenson R, White D, Darbes L, Hoff C, Sullivan P. HIV testing behaviors and perceptions of risk of HIV infection among MSM with main partners. AIDS and Behavior 2015; 19(3): 553-60. doi:10.1007/s10461-014-0862-4.

${ }^{26}$ Jaspal R, Lopes B, Jamal Z, Yap C, Paccoud I, Sekhon P. HIV knowledge, sexual health and behaviour among black and minority ethnic men who have sex with men in the UK: a crosssectional study. Sexual Health 2019; 16(1): 25-31. doi:10.1071/SH18032.

${ }^{27}$ Mao L, Adam P, Kippax S, Holt M, Prestage G, Calmette Y, Zablotska I, de Wit J. HIV-negative gay men's perceived HIV risk hierarchy: imaginary or real? AIDS and Behavior 2013; 17(4): 13629. doi:10.1007/s10461-012-0406-8.

${ }^{28}$ MacKellar DA, Valleroy LA, Secura GM, Behel S, Bingham T, Celentano DD, Koblin BA, Lalota M, Shehan D, Thiede H, Torian LV., Young Men's Survey Study Group. Perceptions of lifetime risk and actual risk for acquiring HIV among young men who have sex with men. AIDS and Behavior 2007, 11(2), 263-70. doi:10.1007/s10461-006-9136-0

${ }^{29}$ Sarno EL, Mohr JJ. Partner Attractiveness and Perceived Sexually Transmitted Infection Risk Among Sexual Minority Men. J Sex Res. 2020;57(5):559-569.

doi:10.1080/00224499.2019.1591335

${ }^{30}$ Napper LE, Fisher DG., Reynolds GL. Development of the perceived risk of HIV scale. AIDS and Behavior 2012; 16(4): 1075-1083. doi:10.1007/s 10461-011-0003-2.

${ }^{31}$ Mohr JJ, Kendra MS. Revision and extension of a multidimensional measure of sexual minority identity: The Lesbian, Gay, and Bisexual Identity Scale. Journal of Counseling Psychology 2011; 58(2): 234-245. doi:10.1037/a0022858

32 Diener E, Emmons RA, Larsen RJ, Griffin S. The Satisfaction With Life Scale. Journal of Personality Assessment 1985; 49(1): 71-75. doi:10.1207/s15327752jpa4901_13

33 Jaspal R, Cinnirella M. Coping with potentially incompatible identities: accounts of religious, ethnic and sexual identities from British Pakistani men who identify as Muslim and gay. British Journal of Social Psychology 2010; 49(4): 849-870. doi:10.1348/014466609X485025

${ }^{34}$ Goldman BM, Kernis MH. The role of authenticity in healthy psychological functioning and subjective well-being. Annals of the American Psychotherapy Association 2002; 5(6): 18-20.

${ }^{35}$ Harper GW, Serrano PA, Bruce D, Bauermeister JA. The Internet's multiple roles in facilitating the sexual orientation identity development of gay and bisexual male adolescents. American Journal of Men's Health 2016; 10(5): 359-76. doi:10.1177/1557988314566227

36 Jaspal R. Sexuality, migration and identity among gay Iranian migrants to the UK. In Taylor Y, Snowdon R, editors. Queering religion, religious queers. London: Routledge; 2014. pp. 44-60 
${ }^{37}$ Fiske ST, Taylor SE. Social cognition (2nd ed.). New York: Mcgraw-Hill Book Company; 1991. ${ }^{38}$ Dubé N, MacDonald E. Vaccine acceptance: barriers, perceived risks, benefits, and irrational beliefs. In Bloom BR, Lambert P, editors. The vaccine book (Second Edition). Cambridge: Academic Press; 2016. pp. 507-528

${ }^{39}$ Breakwell GM. Models of risk construction: some applications to climate change. Wiley Interdisciplinary Reviews: Climate Change 2010; 1(6): 857-870.

${ }^{40}$ Fraser B, Pierse N, Chisholm E, Cook H. LGBTIQ+ homelessness: a review of the literature. International Journal of Environmental Research and Public Health 2019; 16(15): 2677. doi:10.3390/ijerph16152677.

${ }^{41}$ Maatouk I, Jaspal R. HIV in men who have sex with men in Lebanon: clinical and psychosocial aspects. BMJ Sexual \& Reproductive Health 2019; 45: 175-176.

42 Pachankis JE, Hatzenbuehler ML, Hickson F, Weatherburn P, Berg RC, Marcus U, Schmidt AJ. Hidden from health: structural stigma, sexual orientation concealment, and HIV across 38 countries in the European MSM Internet Survey. AIDS 2015; 29(10): 1239-46. 